\title{
Short coherent control pulse with small random errors in its direction
}

\author{
Su Zhi-Kun and Jiang Shao-Ji \\ State Key Laboratory of Optoelectronic Materials and Technologies, Sun Yat-sen \\ University, Guangzhou 510275, People's Republic of China \\ E-mail: stsjsj@mail.sysu.edu.cn
}

\begin{abstract}
The finite-amplitude short coherent control pulse with small random errors in its direction is considered. We derive the conditions for it to approximate an ideal $\delta$-shaped pulse up to an error in the second order of pulse duration. Whether the symmetric and asymmetric pulse shapes would fulfill these conditions is also analyzed. The result shows that, under one of these conditions, all symmetric pulse shapes can fulfill this condition but not for all asymmetric ones.
\end{abstract}




\section{Introduction}

Suppressing decoherence is significantly important for quantum information processing (QIP) and other quantum-based technologies. One of the most effective techniques to combat with the decoherence process is dynamical decoupling (DD) [1,2], which evolves from the Hahn echo [3] and develops for refocusing techniques in nuclear magnetic resonance (NMR) [4] 6]. The basic idea of DD [7,9] is to insert a sequence of short coherent control pulses within a time period of interest, such that the system of interest can be effectively decoupled from its environment. In the theoretical treatments the control pulse is considered as an ideal, i.e. $\delta$-shaped $\pi$ pulse (so-called "bang-bang" or unbounded control) which means that its amplitude is infinite in the sense of a $\delta$ function. This assumption is convenient because all the Hamiltonian of the system can be neglected during the control pulse duration (infinitely short). Hence it is relatively straightforward to cope with the time evolution.

In experiments, however, the ideal instantaneous pulse is not realistic [10,11] since the pulse would have a finite duration $\tau_{p}$ or it might be imperfect, e.g., one can have pulse length or amplitude errors (leading to a wrong angle of rotation) or an off-resonance error, due to which the rotation occurs around a tilted axis. The accumulation effect of these small imperfections in the pulse may have significant consequences when the number of pulses in a DD pulse sequence is not small. The issue of finite duration and finite amplitude has been considered in [12 15]. To mitigate the effect of finite duration and finite amplitude, pulse shaping has been addressed to approximate an instantaneous pulse about a fixed duration [12]. Following the idea developed in [12], the case of a fixed axis has been extended to the case of an axis varying in time [13]. Further numerical work illustrates that tailoring the pulse, including symmetric and asymmetric, improves the quality of a real pulse significantly [14]. But the small deviations of the actual direction of the pulse have not been considered in [12 15].

On the other hand, the effect of small random errors in the direction has been studied in a comprehensive comparison of various DD sequence in [16, 17]. However, the $\pi$ pulse considered in [16, 17] is instantaneous and its amplitude is infinite. Hence the discussion is given in the absence of the coupling to the bath during the pulse and on the level of pulse sequences. In this paper, we consider a finite-amplitude pulse with random errors in its directions on the level of an individual pulse. We aim to provide some guidelines in the choice of pulse shapes, i.e. symmetric and asymmetric shapes, when the rotation occurs around a tilted axis. To realize the goal, we follow the idea proposed in [15] and derive the expressions of error terms for this finite-amplitude pulse to simulate an ideal pulse up to an error of $O\left(\tau_{p}^{2}\right)$ in the presence of small random errors in its direction, where $\tau_{p}$ is the pulse duration. We divide these terms into two groups, those from pulse duration only and the others originate from errors in pulse direction, and discuss them separately. As expected, both symmetric and asymmetric pulse shapes can make the error terms from pulse duration vanish. However, neither type of the pulse can remove the error terms from pulse direction completely, but all symmetric pulse can 
make one more terms equal zero than the specific choice of asymmetric one. Our results can be useful for experimental implementations of kinds of DD protocols, and for deeper understanding of a short coherent control pulse which is subject to random errors around its direction.

The paper is organized as follows. After introducing the model (section 2), we start discussing the error terms due to pulse duration and reviewing two kinds of optimized pulse shapes which are designed to eliminate these terms (section 3). Then the error terms coming from deviation in rotation axis are analyzed (section 4). At last the conclusion is given (section 5 ).

\section{Model}

The total Hamiltonian of system, bath and the interaction between them is given by $H$. To suppress decoherence, we implement fast control on the system and the control Hamiltonian reads

$$
H_{\Omega}(t)=V(t) \Omega
$$

where $V(t)$ stands for the pulse shape as a function of time and $\Omega$ is a parity kick operator, i.e., $\Omega \Omega^{\dagger}=\Omega^{2}=1$ [18]. In reality, the controlling pulses may be subject to small errors in its direction, so that the the Hamiltonian $H_{\Omega}(t)$ in Eq. (11) should be corrected into

$$
H_{C}(t)=V(t)\left(\Omega+\epsilon \Omega^{\prime}\right)
$$

where $\epsilon$ is the small error factor and $\Omega^{\prime}$ is an arbitrary operator of the system. The evolution operator during the pulse of length $\tau_{p}$ takes the form of

$$
\begin{aligned}
U\left(\tau_{p}, 0\right) & =\overleftarrow{T} \exp \left\{-i \int_{0}^{\tau_{p}}\left[H+H_{C}(t)\right] d t\right\} \\
& =e^{-i H\left(\tau_{p}-\tau_{s}\right)} U_{C} e^{-i H \tau_{s}}
\end{aligned}
$$

where $\overleftarrow{T}$ stands for the time-ordering operator with time increasing from right to left, $\tau_{s}$ is an arbitrary instant inside the interval $\left[0, \tau_{p}\right]$ (more detail about will be given below Eq.(미) $)$, and

$$
U_{C}=\overleftarrow{T} \exp \left[-i \int_{0}^{\tau_{p}}\left[\widetilde{H}_{C}(t)\right] d t\right]
$$

with $\widetilde{H}_{C}(t)=e^{i H\left(t-\tau_{s}\right)} H_{C}(t) e^{-i H\left(t-\tau_{s}\right)}$. On the other hand, the evolution, described by Eq.(3), can be rewritten as

$$
\begin{aligned}
U\left(\tau_{p}, 0\right) & =\overleftarrow{T} \exp \left\{-i \int_{0}^{\tau_{p}}\left[H+H_{C}(t)\right] d t\right\} \\
& =e^{-i H\left(\tau_{p}-\tau_{s}\right)} P_{\Omega} e^{-i H \tau_{s}}+O(\epsilon)+O\left(\epsilon \tau_{p}\right)+O\left(\tau_{p}^{2}\right)
\end{aligned}
$$


Short coherent control pulse with small random errors in its direction

where $P_{\Omega}=\exp \left[-i \int_{0}^{\tau_{p}} H_{\Omega}(t) d t\right]$ is the desired instantaneous control applied at the time $\tau_{s}$. Next, we follow the method outlined in [15] to find out the expressions of the error terms $O(\epsilon)+O\left(\epsilon \tau_{p}\right)$ in the last line of Eq.(5). Compare $U_{C}$ in Eq.(3) with $P_{\Omega}$ in Eq.(5), we evaluate first the error in the control Hamiltonian

$$
\begin{aligned}
h_{\Omega}(t) & =\widetilde{H}_{C}(t)-H_{\Omega} \\
& \left.=V(t)\left\{\sum_{k=1}^{\infty} \frac{\left(t-\tau_{s}\right)^{k}}{k !}\left[(i H)^{k}, \Omega\right]+\epsilon \Omega^{\prime}+\epsilon \sum_{k=1}^{\infty} \frac{\left(t-\tau_{s}\right)^{k}}{k !}\left[(i H)^{(k)}, \Omega^{\prime}\right]\right\}\right)
\end{aligned}
$$

The notation stands for

$$
\begin{aligned}
& {\left[(i H)^{(1)}, \cdots\right]=[i H, \cdots]} \\
& {\left[(i H)^{(2)}, \cdots\right]=[i H,[i H, \cdots]],} \\
& {\left[(i H)^{(3)}, \cdots\right]=[i H,[i H,[i H, \cdots]]],}
\end{aligned}
$$

and so on. Now we have the error in the evolution

$$
\begin{aligned}
\delta P_{\Omega} & \equiv U_{C}-P_{\Omega} \\
& =\overleftarrow{T}\left\{e^{-i \int_{0}^{\tau_{p}} H_{\Omega}(t) d t}\left[e^{-i \int_{0}^{\tau_{p}} h_{\Omega}(t) d t}-1\right]\right\} .
\end{aligned}
$$

Then the leading-order in $\delta P_{\Omega}$, i.e. to the first order in $\tau_{p} H$ and $\epsilon$, is [19]

$$
\begin{aligned}
\eta & =\overleftarrow{T}\left\{e^{-i \int_{0}^{\tau_{p}} H_{\Omega}(t) d t} \int_{0}^{\tau_{p}} V(t)\left\{\left(t-\tau_{s}\right)[H, \Omega]-i \epsilon \Omega^{\prime}+\left(t-\tau_{s}\right) \epsilon\left[H, \Omega^{\prime}\right]\right\} d t\right\} \\
& =\int_{0}^{\tau_{p}} V(t) e^{-i \int_{t}^{\tau_{p}} H_{\Omega}(s) d s}\left\{-i \epsilon \Omega^{\prime}+\left(t-\tau_{s}\right) \epsilon\left[H, \Omega^{\prime}\right]+\left(t-\tau_{s}\right)[H, \Omega]\right\} e^{-i \int_{0}^{t} H_{\Omega}(s) d s} d t \\
& =\eta^{(\epsilon, 1)\left(\tau_{p}, 0\right)}+\eta^{(\epsilon, 1)\left(\tau_{p}, 1\right)}+\eta^{\left(\tau_{p}, 1\right)},
\end{aligned}
$$

where the superscript $(u, v)$ of $\eta$ in the last line of Eq.(11) denotes an error in the $v$ th order of $u$. Take the superscript $\eta^{(\epsilon, 1)\left(\tau_{p}, 1\right)}$ for an example, this term means an error in the first order of $\epsilon$ and an error in the first order of $\tau_{p}$ as well. Here we have defined

$$
\begin{aligned}
& \eta^{(\epsilon, 1)\left(\tau_{p}, 0\right)}=-i \epsilon \int_{0}^{\tau_{p}} V(t) e^{-i \int_{t}^{\tau_{p}} H_{\Omega}(s) d s} \Omega^{\prime} e^{-i \int_{0}^{t} H_{\Omega}(s) d s} d t, \\
& \eta^{(\epsilon, 1)\left(\tau_{p}, 1\right)}=\epsilon \int_{0}^{\tau_{p}}\left(t-\tau_{s}\right) V(t) e^{-i \int_{t}^{\tau_{p}} H_{\Omega}(s) d s}\left[H, \Omega^{\prime}\right] e^{-i \int_{0}^{t} H_{\Omega}(s) d s} d t,
\end{aligned}
$$

and

$$
\eta^{\left(\tau_{p}, 1\right)}=\int_{0}^{\tau_{p}}\left(t-\tau_{s}\right) V(t) e^{-i \int_{t}^{\tau_{p}} H_{\Omega}(s) d s}[H, \Omega] e^{-i \int_{0}^{t} H_{\Omega}(s) d s} d t .
$$

Note that the term described by Eq.(14) is the same as those derived in [12, 15]. The error $\eta^{\left(\tau_{p}, 1\right)}$ does not come from directional error and it can be eliminated using pulse shaping [12 14]. In the following two sections, we will analyze how shaped pulses reduce the errors $\eta^{\left(\tau_{p}, 1\right)}, \eta^{(\epsilon, 1)\left(\tau_{p}, 0\right)}$, and $\eta^{(\epsilon, 1)\left(\tau_{p}, 1\right)}$, respectively, and with which types of pulse shape the errors will approximate as close as possible to zero. Furthermore, we 
should mention that, the model discussed in this section is general, for example, we can take $\Omega \rightarrow \sigma_{x}$ and $\epsilon \Omega^{\prime} \rightarrow \epsilon_{y} \sigma_{y}+\epsilon_{z} \sigma_{z}$ for a single-qubit system and the control pulse corresponds to a rotation about $x$ axis with small deviation $\epsilon_{y}$ and $\epsilon_{z}$ in the $y$ and $z$ direction, respectively, where $\sigma_{x}, \sigma_{y}$ and $\sigma_{z}$ are the standard Pauli matrices.

\section{Error due to pulse duration $\left(\eta^{\left(\tau_{p}, 1\right)}\right)$}

In this section, the expression of $\eta^{\left(\tau_{p}, 1\right)}$ is given, then the symmetry and asymmetric pulse shapes, which are designed to eliminate $\eta^{\left(\tau_{p}, 1\right)}$, are also introduced.

We begin to calculate the expression. Using $\Omega^{2}=1$ and Eq.(1), we have

$$
e^{-i \int_{t_{1}}^{t_{2}} H_{\Omega}(s) d s}=\cos \left[\int_{t_{1}}^{t_{2}} V(s) d s\right]-i \Omega \sin \left[\int_{t_{1}}^{t_{2}} V(s) d s\right] .
$$

The total Hamiltonian $H$ can be decomposed into two parts [15]

$$
H=H_{a}+H_{c}
$$

with $H_{a}$ and $H_{c}$ anticommuting and commuting with $\Omega$, respectively. Then from Eq.(14) one has

$$
\eta^{\left(\tau_{p}, 1\right)}=i 2 H_{a} \eta_{1}^{\left(\tau_{p}, 1\right)}+2 H_{a} \Omega \eta_{2}^{\left(\tau_{p}, 1\right)}
$$

where

$$
\begin{aligned}
& \eta_{1}^{\left(\tau_{p}, 1\right)}=\int_{0}^{\tau_{p}}\left(t-\tau_{s}\right) V(t) \sin \left[\phi_{-}-\psi(t)\right] d t, \\
& \eta_{2}^{\left(\tau_{p}, 1\right)}=\int_{0}^{\tau_{p}}\left(t-\tau_{s}\right) V(t) \cos \left[\phi_{-}-\psi(t)\right] d t,
\end{aligned}
$$

with $\psi(t) \equiv 2 \int_{\tau_{s}}^{t} V(s) d s$ and $\phi_{ \pm}=\int_{\tau_{s}}^{\tau_{p}} V(s) d s \pm \int_{0}^{\tau_{s}} V(s) d s$ ( $\phi_{+}$will be used later). To make $\eta_{1}^{\left(\tau_{p}, 1\right)}=\eta_{2}^{\left(\tau_{p}, 1\right)}=0$, two kinds of composite pulses, which consist of piecewise constant pulses of maximally positive or negative amplitude $\pm a_{\max }$, have been designed in [12 14]. Here we review so-called symmetric pulses first and then asymmetric pulses.

For symmetric pulses with $\tau_{s}=\tau_{p} / 2$, one needs at least two free parameters including $\tau_{s}$, in order to obtain pulses which show only quadratic deviations from the idealized instantaneous pulse. One way to approach the solution of the one additional free parameter is to take the instant $\tau_{1}$, at which the pulse changes first and then another sign change occurs by symmetry at $t=\tau_{p}-\tau_{1}$. The pulse shape $V(t)$ can be depicted as

$$
V_{s}(t)=\left\{\begin{array}{ccc}
a_{\max } & \text { if } & \tau_{1} \leq t \leq \tau_{p}-\tau_{1} \\
-a_{\max } & \text { if } & 0 \leq t<\tau_{1}
\end{array} \quad \text { or } \quad \tau_{p}-\tau_{1}<t \leq \tau_{p} .\right.
$$


Short coherent control pulse with small random errors in its direction

For asymmetric pulses case, on the other hand, the composite pulse consists of two constant regions only. The pulse shape gives

$$
V_{a}(t)=\left\{\begin{array}{ccc}
a_{\max } & \text { if } & 0 \leq t<\tau_{1} \\
-a_{\max } & \text { if } & \tau_{1} \leq t \leq \tau_{p}
\end{array} .\right.
$$

It is noteworthy that the value of every parameter, i.e. $\tau_{s}, \tau_{1}$ and $\pm a_{\max }$, are determined by the intended angle of rotation of the pulse besides Eq.(18) and Eq.(19). In addition, the pulse shapes described by Eq.(20) and Eq.(21) are just two of the solutions discussed in [12-14]. In the next section, we will study which type of pulse, symmetric or asymmetric, is more suitable for the experimental situation under consideration the small random errors in the rotation direction.

\section{Error due to rotation axis $\left(\eta^{(\epsilon, 1)\left(\tau_{p}, 0\right)}\right.$ and $\left.\eta^{(\epsilon, 1)\left(\tau_{p}, 1\right)}\right)$}

In this section, we consider both $\eta^{(\epsilon, 1)\left(\tau_{p}, 0\right)}$ and $\eta^{(\epsilon, 1)\left(\tau_{p}, 1\right)}$, which are not studied in [15]. For short time $\tau_{p}$, one has $\eta^{(\epsilon, 1)\left(\tau_{p}, 0\right)}>\eta^{(\epsilon, 1)\left(\tau_{p}, 1\right)}$. Let us first consider the dominant error $\eta^{(\epsilon, 1)\left(\tau_{p}, 0\right)}$ in the error due to rotation axis. As in section 3 , we separate the arbitrary operator of the system $\Omega^{\prime}$ into two parts

$$
\Omega^{\prime}=\Omega_{a}^{\prime}+\Omega_{c}^{\prime},
$$

with $\Omega_{a}^{\prime}$ and $\Omega_{c}^{\prime}$ anticommuting and commuting with $\Omega$, respectively. Hence $\eta^{(\epsilon, 1)\left(\tau_{p}, 0\right)}$ in Eq.(12) takes the form of

$$
\eta^{(\epsilon, 1)\left(\tau_{p}, 0\right)}=-\Omega \Omega_{a}^{\prime} \eta_{1}^{(\epsilon, 1)\left(\tau_{p}, 0\right)}-i \Omega_{a}^{\prime} \eta_{2}^{(\epsilon, 1)\left(\tau_{p}, 0\right)}-\Omega \Omega_{c}^{\prime} \eta_{3}^{(\epsilon, 1)\left(\tau_{p}, 0\right)}-i \Omega_{c}^{\prime} \eta_{4}^{(\epsilon, 1)\left(\tau_{p}, 0\right)},
$$

where

$$
\begin{aligned}
\eta_{1}^{(\epsilon, 1)\left(\tau_{p}, 0\right)} & =\int_{0}^{\tau_{p}} \epsilon V(t) \sin \left[\phi_{-}-\psi(t)\right] d t \\
\eta_{2}^{(\epsilon, 1)\left(\tau_{p}, 0\right)} & =\int_{0}^{\tau_{p}} \epsilon V(t) \cos \left[\phi_{-}-\psi(t)\right] d t \\
\eta_{3}^{(\epsilon, 1)\left(\tau_{p}, 0\right)} & =\int_{0}^{\tau_{p}} \epsilon V(t) \sin \left[\phi_{+}\right] d t \\
\eta_{4}^{(\epsilon, 1)\left(\tau_{p}, 0\right)} & =\int_{0}^{\tau_{p}} \epsilon V(t) \cos \left[\phi_{+}\right] d t
\end{aligned}
$$

with $\phi_{+}$defined just below Eq.(19). It is worth to mention that the value of $\phi_{+}$equals half of the intended angle of rotation of the pulse, such as $\phi_{+}=\pi / 2$ for the $\pi$ pulse and $\phi_{+}=\pi / 4$ for the $\pi / 2$ pulse. Before discussing how pulse shaping reduces the errors described by Eq.(24) $\sim$ Eq.(27), we derive the expressions of $\phi_{-}-\psi(t)$ for general symmetric and asymmetric pulse shapes

$$
\phi_{-}-\psi(t)=\frac{\pi}{2}-2 \int_{0}^{t} V(s) d s,
$$


Short coherent control pulse with small random errors in its direction

Hence we have

$$
\begin{aligned}
\eta_{1}^{(\epsilon, 1)\left(\tau_{p}, 0\right)} & =\epsilon \int_{0}^{\tau_{p}} V(t) \cos \left[2 \int_{0}^{t} V(s) d s\right] d t \\
& =\frac{\epsilon}{2} \int_{0}^{\tau_{p}} \cos \left[2 \int_{0}^{t} V(s) d s\right] d\left[2 \int_{0}^{t} V(s) d s\right] \\
& =0 \\
\eta_{2}^{(\epsilon, 1)\left(\tau_{p}, 0\right)} & =\epsilon \int_{0}^{\tau_{p}} V(t) \sin \left[2 \int_{0}^{t} V(s) d s\right] d t \\
& =\frac{\epsilon}{2} \int_{0}^{\tau_{p}} \sin \left[2 \int_{0}^{t} V(s) d s\right] d\left[2 \int_{0}^{t} V(s) d s\right] \\
& =\epsilon, \\
\eta_{3}^{(\epsilon, 1)\left(\tau_{p}, 0\right)} & =\frac{\pi}{2} \epsilon, \\
\eta_{4}^{(\epsilon, 1)\left(\tau_{p}, 0\right)} & =0,
\end{aligned}
$$

for both symmetric and asymmetric pulses in terms of the $\pi$ pulse, i.e. $\Phi_{s}=\Phi_{a}=\pi$. So both pulse shapes have the same effect on Eq.(24) ${ }^{\sim}$ Eq.(27). Besides, we have $\frac{1}{\epsilon} \eta_{2}^{(\epsilon, 1)\left(\tau_{p}, 0\right)} \neq 0$ and $\eta_{3}^{(\epsilon, 1)\left(\tau_{p}, 0\right)} \neq 0$ no matter what type of composite pulse ia applied to optimize the coherent control pulse. From this point of view the impact of errors in the direction of the rotation axis cannot be ignored completely.

Now, the analogous procedure is used to obtain

$$
\begin{aligned}
\eta^{(\epsilon, 1)\left(\tau_{p}, 1\right)}= & -i \Omega\left[\left(H_{a} \Omega_{a}^{\prime}-\Omega_{a}^{\prime} H_{a}\right)+\left(H_{c} \Omega_{c}^{\prime}-\Omega_{c}^{\prime} H_{c}\right)\right] \eta_{1}^{(\epsilon, 1)\left(\tau_{p}, 1\right)} \\
& +\left[\left(H_{a} \Omega_{a}^{\prime}-\Omega_{a}^{\prime} H_{a}\right)+\left(H_{c} \Omega_{c}^{\prime}-\Omega_{c}^{\prime} H_{c}\right)\right] \eta_{2}^{(\epsilon, 1)\left(\tau_{p}, 1\right)} \\
& -i \Omega 2\left(H_{a} \Omega_{c}^{\prime}+H_{c} \Omega_{a}^{\prime}\right) \eta_{3}^{(\epsilon, 1)\left(\tau_{p}, 1\right)}+2\left(H_{a} \Omega_{c}^{\prime}+H_{c} \Omega_{a}^{\prime}\right) \eta_{4}^{(\epsilon, 1)\left(\tau_{\varphi}(3),\right)}
\end{aligned}
$$

where

$$
\begin{aligned}
& \eta_{1}^{(\epsilon, 1)\left(\tau_{p}, 1\right)}=\epsilon \int_{0}^{\tau_{p}}\left(t-\tau_{s}\right) V(t) \sin \left[\phi_{+}\right] d t, \\
& \eta_{2}^{(\epsilon, 1)\left(\tau_{p}, 1\right)}=\epsilon \int_{0}^{\tau_{p}}\left(t-\tau_{s}\right) V(t) \cos \left[\phi_{+}\right] d t, \\
& \eta_{3}^{(\epsilon, 1)\left(\tau_{p}, 1\right)}=\epsilon \int_{0}^{\tau_{p}}\left(t-\tau_{s}\right) V(t) \sin \left[\phi_{-}-\psi(t)\right] d t, \\
& \eta_{4}^{(\epsilon, 1)\left(\tau_{p}, 1\right)}=\epsilon \int_{0}^{\tau_{p}}\left(t-\tau_{s}\right) V(t) \cos \left[\phi_{-}-\psi(t)\right] d t,
\end{aligned}
$$

Hence, we find that both symmetric and asymmetric pulses (see Eq.(20) and Eq.(21)) can make terms $\eta_{3}^{(\epsilon, 1)\left(\tau_{p}, 1\right)}$ and $\eta_{4}^{(\epsilon, 1)\left(\tau_{p}, 1\right)}$ vanish. In addition, we obtain $\eta_{2}^{(\epsilon, 1)\left(\tau_{p}, 1\right)}=0$ for the $\pi$ pulse, i.e. $\phi_{+}=\pi / 2$. Next, we look at Eq.(34). For symmetric pulse shape, Eq.(34) gives 
Short coherent control pulse with small random errors in its direction

Table 1. The comparison between symmetric pulse (SP) and asymmetric pulse (AP) for all terms discussed in section 3. According to the additional condition (AC) and results, the terms have been divided into 4 groups. With the additional condition, we will have the result, " $=0$ " or " $\neq 0$ ", for the terms. The result " $=0$ " or " $\neq 0$ " means that the corresponding term is zero or nonzero. The additional condition "no AC" means there is no additional condition. " $\Phi=\pi$ " implies that the pulse considered here is a $\pi$ pulse. And " $\triangle$ " indicates that we have the corresponding results for all symmetric pulse and just for the specific choice of asymmetric pulse.

\begin{tabular}{llll}
\hline Terms & $S P$ & $A P$ & $A C$ \\
\hline$\eta_{1}^{\left(\tau_{p}, 1\right)}, \eta_{2}^{\left(\tau_{p}, 1\right)}, \eta_{3}^{(\epsilon, 1)\left(\tau_{p}, 1\right)}, \eta_{4}^{(\epsilon, 1)\left(\tau_{p}, 1\right)}$ & $=0$ & $=0$ & no AC \\
$\eta_{1}^{(\epsilon, 1)\left(\tau_{p}, 0\right)}, \eta_{4}^{(\epsilon, 1)\left(\tau_{p}, 0\right)}, \eta_{2}^{(\epsilon, 1)\left(\tau_{p}, 1\right)}$ & $=0$ & $=0$ & $\Phi=\pi$ \\
$\eta_{2}^{(\epsilon, 1)\left(\tau_{p}, 0\right)}, \eta_{3}^{(\epsilon, 1)\left(\tau_{p}, 0\right)}$ & $\neq 0$ & $\neq 0$ & $\Phi=\pi$ \\
$\eta_{1}^{(\epsilon, 1)\left(\tau_{p}, 1\right)}$ & $=0$ & $\neq 0$ & $\triangle$ \\
\hline
\end{tabular}

$$
\begin{aligned}
\frac{1}{\epsilon} \eta_{1, s}^{(\epsilon, 1)\left(\tau_{p}, 1\right)} & =\int_{0}^{\tau_{s}}\left(t-\tau_{s}\right) V(t) d t+\int_{\tau_{s}}^{2 \tau_{s}}\left(t-\tau_{s}\right) V(t) d t \\
& =0
\end{aligned}
$$

we have use the relation $V(t)=V\left(2 \tau_{s}-t\right)$ in the last line of Eq.(38). It should be noted that we have $\eta_{1, s}^{(\epsilon, 1)\left(\tau_{p}, 1\right)}=0$ for all symmetric $\pi$ pulse, including the specific shaped pulse given in Eq.(20). For asymmetric pulse shape described by Eq.(21), this error term is in the form of

$$
\frac{1}{\epsilon} \eta_{1, a}^{(\epsilon, 1)\left(\tau_{p}, 1\right)}=a_{\max }\left(\tau_{1}^{2}-\frac{\tau_{p}^{2}}{2}-2 \tau_{1} \tau_{s}+\tau_{p} \tau_{s}\right)
$$

In spite of our intensive search we have not succeeded in proving $\tau_{1}^{2}-\tau_{p}^{2} / 2-2 \tau_{1} \tau_{s}+\tau_{p} \tau_{s} \neq$ 0 . However, we argue that at least some asymmetric pulse shapes cannot make $\eta_{1, a}^{(\epsilon, 1)\left(\tau_{p}, 1\right)}$ vanish. Take $\tau_{1}=(2 n+1) \tau_{p} /(4 n), \tau_{s}=\tau_{p}\left[1 / 2+(-1)^{n} /(2 n \pi)\right]$ and $a_{\max }=\pi n / \tau_{p}$, where $n$ is a positive integer, for instance, we get

$$
\frac{1}{\epsilon} \eta_{1, a}^{(\epsilon, 1)\left(\tau_{p}, 1\right)}=\frac{\left[-4(-1)^{n}+\pi-4 n^{2} \pi\right] \tau_{p}}{16 n} \neq 0 .
$$

Therefore, the symmetric pulse shape eliminates the errors more effectively than the asymmetric one does in terms of Eq. (34) for the specific choice of pulse shapes.

Finally, the results discussed in section 3 and 4 are listed in Table 11. As shown in Table 1, we should note that except the term $\eta_{1}^{(\epsilon, 1)\left(\tau_{p}, 1\right)}$ for the specific choice of asymmetric pulse, the rest results are not dependent on the specific form of pulse shape (given by Eq.(20) and Eq.(21)), that is, we get the rest results from all symmetric or asymmetric pulse. 


\section{Conclusions}

In this paper, we follow the method outlined in [15] to study a finite-amplitude short coherent control pulse with small random errors in its direction. Note that the errors in direction is neglected in [15]. The error $\eta^{\left(\tau_{p}, 1\right)}$, which comes from pulse duration $\tau_{p}$ only, is reviewed first and the optimized pulse shapes for a single-qubit flip control can be used to eliminate this error as expected [12 14]. Secondly, we consider the error terms

$\eta^{(\epsilon, 1)\left(\tau_{p}, 0\right)}$ and $\eta^{(\epsilon, 1)\left(\tau_{p}, 1\right)}$, which originate from deviation in rotation axis. The result shows that the term $\eta^{(\epsilon, 1)\left(\tau_{p}, 0\right)}$ can not be removed completely by either symmetric or asymmetric shaped pulse and both types of pulse have the same effect in view of this term. As for the term $\eta^{(\epsilon, 1)\left(\tau_{p}, 1\right)}$, we find that any symmetric pulse shaping can make it vanish while the specific choice of asymmetric one can not. It should be mentioned that symmetric pulse shape performs better than asymmetric pulse just for the specific pulse shapes which we choose. This work can be useful for experimental implementations of kinds of DD protocols, and for deeper understanding of a short coherent control pulse which is subject to random errors in its directions.

Further work should verify the results in specific models in order to understand better how important the pulse shape in approximating $\delta$-shaped pulse really are. In addition, the more generic situation with pulse errors, e.g. pulse length errors, amplitude errors or an off-resonance error, is much more complex and further investigation is needed.

\section{Acknowledgements}

This work is supported by the National Natural Science Foundation of China under Grant No.60977042.

\section{References}

[1] Lorenza Viola and Seth Lloyd. Dynamical suppression of decoherence in two-state quantum systems. Phys. Rev. A, 58:2733-2744, Oct 1998.

[2] Lorenza Viola, Emanuel Knill, and Seth Lloyd. Dynamical decoupling of open quantum systems. Phys. Rev. Lett., 82:2417-2421, Mar 1999.

[3] E. L. Hahn. Spin echoes. Phys. Rev., 80:580-594, Nov 1950.

[4] W.-K. Rhim, A. Pines, and J. S. Waugh. Violation of the spin-temperature hypothesis. Phys. Rev. Lett., 25:218-220, Jul 1970.

[5] M. Mehring. Principles of high-resolution NMR in solids. Springer-Verlag in Berlin, New York, 1983.

[6] U. Haeberlen. High Resolution NMR in Solids: Selective Averaging. Academic Press, New York, 1976.

[7] Łukasz Cywiński, Roman M. Lutchyn, Cody P. Nave, and S. Das Sarma. How to enhance dephasing time in superconducting qubits. Phys. Rev. B, 77:174509, May 2008.

[8] M. J. Biercuk, A. C. Doherty, and H. Uys. Dynamical decoupling sequence construction as a filterdesign problem. Journal of Physics B: Atomic, Molecular and Optical Physics, 44(15):154002, 2011. 
[9] Zhi-Kun Su and Shao-Ji Jiang. Filter-design perspective applied to dynamical decoupling of a multi-qubit system. Journal of Physics B: Atomic, Molecular and Optical Physics, 45(2):025502, 2012.

[10] Michael J. Biercuk, Hermann Uys, Aaron P. VanDevender, Nobuyasu Shiga, Wayne M. Itano, and John J. Bollinger. Optimized dynamical decoupling in a model quantum memory. Nature, 458:996, 2009.

[11] Gonzalo A. Álvarez, Ashok Ajoy, Xinhua Peng, and Dieter Suter. Performance comparison of dynamical decoupling sequences for a qubit in a rapidly fluctuating spin bath. Phys. Rev. A, 82:042306, Oct 2010.

[12] S. Pasini, T. Fischer, P. Karbach, and G. S. Uhrig. Optimization of short coherent control pulses. Phys. Rev. A, 77:032315, Mar 2008.

[13] S Pasini and G S Uhrig. Generalization of short coherent control pulses: extension to arbitrary rotations. Journal of Physics A: Mathematical and Theoretical, 41(31):312005, 2008.

[14] P. Karbach, S. Pasini, and G. S. Uhrig. Numerical analysis of optimized coherent control pulses. Phys. Rev. A, 78:022315, Aug 2008.

[15] Zhen-Yu Wang and Ren-Bao Liu. Protection of quantum systems by nested dynamical decoupling. Phys. Rev. A, 83:022306, Feb 2011.

[16] Zhihao Xiao, Lewei He, and Wen-ge Wang. Efficiency of dynamical decoupling sequences in the presence of pulse errors. Phys. Rev. A, 83:032322, Mar 2011.

[17] Zhi-Hui Wang and V V Dobrovitski. Aperiodic dynamical decoupling sequences in the presence of pulse errors. Journal of Physics B: Atomic, Molecular and Optical Physics, 44(15):154004, 2011.

[18] D. Vitali and P. Tombesi. Using parity kicks for decoherence control. Phys. Rev. A, 59:4178-4186, Jun 1999.

[19] Richard P. Feynman. An operator calculus having applications in quantum electrodynamics. Phys. Rev., 84:108-128, Oct 1951. 